\title{
La deixis en français dans une perspective multimodale
}

\section{Deixis in French from a Multimodal Perspective}

Jérôme Jacquin

Université de Lausanne

\section{POURQUOI REVENIR SUR LA DEIXIS ?}

En première analyse, la deixis constitue un sous-ensemble d'unités linguistiques particulièrement stable et bien documenté dans une large diversité de langues ${ }^{1}$. Les « déictiques » sont classiquement définis comme les unités qui disposent de "variables contextuelles inhérentes » (Levinson, 2006 : 107, ma traduction), c'est-à-dire des unités dont le sémantisme intègre une variable relative à la situation - personnelle, spatiale ou temporelle - de communication. Ainsi, à la différence de MAISON, il est impossible de proposer une définition de JE qui ne contienne en son sein une information de type " personne qui parle ». Non seulement les déictiques pointent-

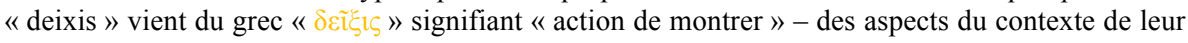
utilisation (Fillmore, 1975 ; Lyons, 1977), mais ils constituent également des « embrayeurs » ou «shifters» dans la mesure où ils changent de référence en fonction de ce même contexte (Jakobson, 1957 ; Jespersen, 1922). En tant qu'elle interroge le rapport des unités linguistiques à leur contexte immédiat de production, la deixis a très tôt intégré le programme de recherche de la Pragmatique (Morris, 1938). Plus récemment, et sous l'impulsion d'une perspective moins déterministe et plus dynamique sur le contexte (Duranti \& Goodwin, 1992), la deixis et les mécanismes de co-orientation et d'attention conjointe sur lesquelles elle repose ont reçu une place de choix dans l'interactionnisme au sens large (e.g. Auer, 1988; Goodwin, 1987 ; Hausendorff, 1995).

Parallèlement, ces quinze dernières années témoignent d'une multiplication de travaux consacrés à la multimodalité. On entend généralement par ce terme le fait que les unités linguistiques et les procédés discursifs intègrent et prennent sens dans des comportements signifiants plus larges, notamment par leur articulation avec la corporalité, qu'il s'agisse des phénomènes prosodiques, des mouvements mimo-faciaux, des gestes, postures et manipulations d'artefacts (y compris de documents) ou encore de la répartition des corps dans l'espace (e.g. Deppermann, 2013 ; Fricke, 2012a ; Jewitt, 2009; Kress \& Van Leeuwen, 2001 ; Müller et al.,

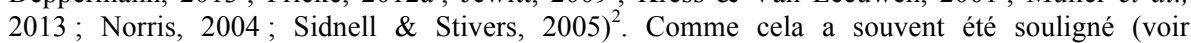

\footnotetext{
${ }^{1}$ Sans recherche d'exhaustivité, on peut citer diverses monographies ou volumes collectifs généralistes (Fillmore, 1975 ; Lenz, 2003 ; Morel \& Danon-Boileau, 1992 ; Tanaka, 2011), comparatistes, translinguistiques ou ciblant une langue particulière (Almeida, 2000 ; Ehrich, 1992 ; Fricke, 2012b ; Jeppesen Kragh \& Lindschouw, 2013 ; Perkins, 1992 ; Senft, 2004) ou encore appliqués à des types de corpus spécifiques (Schmauks, 1991 ; Stukenbrock, 2015).

${ }^{2}$ « Mode is a socially shaped and culturally given resource for making meaning. Image, writing, layout, music, gesture, speech, moving image, soundtrack are examples of modes used in representation and communication » (Kress, 2009 : 54). La multimodalité renvoie ainsi plus généralement à la pluralité des systèmes de signes, et par conséquent à la potentielle diversité de canaux sensoriels (auditif, visuel, olfactif, tactile, gustatif) mobilisés dans l'acte de communication. Dans cette perspective, une page de
} 
notamment Jones \& LeBaron, 2002), rendre justice à la complexité sémiotique des pratiques ordinaires invite non seulement à s'extraire d'un verbo-centrisme régulièrement critiqué, mais aussi à reconsidérer la forme, la valeur et le fonctionnement des unités linguistiques, qui sont dès lors envisagées comme des ressources, parmi d'autres, pour la communication et l'interaction sociale.

L'analyse de la deixis a bénéficié de cette réflexion sur la multimodalité de la parole-eninteraction comprise comme environnement sémiotique naturel des formes verbales. Mais cet effort d'intégration a surtout été conduit à partir de données anglophones et germanophones. Le but et l'originalité du présent volume résident par conséquent dans le fait de tirer les conséquences théoriques, analytiques et descriptives d'appréhender la deixis en français à partir d'une approche multimodale de la référence, telle qu'elle s'accomplit dans l'interaction sociale. Il s'agit plus concrètement de réinterroger localement des unités classiques de la deixis à partir de données vidéo-enregistrées documentant différents contextes interactionnels et multimodaux.

La Section 2 revient sur la délicate question de l'extension du phénomène soumis ici à l'analyse, tandis que la Section 3 retrace à grands traits le destin de la notion de deixis en linguistique française. La Section 4 dégage l'intérêt et les défis représentés par l'adoption d'une perspective multimodale sur la deixis dans l'interaction en face à face. La Section 5 présente finalement le volume et la manière dont les contributions s'articulent en son sein.

\section{INDEXICALITE ET DEIXIS EXOPHORIQUE}

Il faut apporter maintenant deux précisions relatives à l'extension du phénomène soumis à investigation dans le cadre du présent volume. Alors que la première précision revient à différencier l'indexicalité comme principe général et la deixis comme sous-système linguistique à fonctionnement indexical, la seconde concerne les paramètres contextuels retenus et par conséquent le type de deixis considéré ici.

(i) Un déictique comme JE, à la différence d'un terme comme MAISON, repose sur un fonctionnement indexical (ou indiciel) au sens de Peirce (1932) dans la mesure où il fait intervenir un «rapport "naturel" de cooccurrence, de contiguïté existentielle» (Berrendonner, 1981 : 217-218) entre le signe et le référent: JE est contigu à son référent, tout comme la fumée l'est au feu qui en est à l'origine ${ }^{3}$. Ce principe d'indexicalité comme manière particulière de penser la relation entre le signe et son contexte a été fortement généralisé sous l'impulsion de l'ethnométhodologie (Garfinkel, 1967 ; Suchman, 1987) et de l'analyse conversationnelle à sa suite (voir notamment les chapitres réunis dans Sidnell \& Stivers, 2013). Ces traditions considèrent en effet que toutes les actions sociales et toutes les formes linguistiques sont indexicales dans la mesure où leur sens est indexé sur les circonstances de leur production (Duranti \& Goodwin, 1992). Ainsi introduite, une telle perspective risquerait de rendre le présent projet en partie caduc. Il nous faut toutefois - et avec Levinson (2006) - être plus précis: si toutes les unités, une fois mobilisées en contexte, sont indexicales, cela ne signifie pas pour autant que toutes les unités disposent, dans leur sens même, de «variables contextuelles inhérentes » ou, dit autrement, qu'elles « invoquent nécessairement des composantes du contexte en raison de la variable contextuelle qui se trouve encapsulée dans leurs conditions sémantiques » (ibid. : 107, ma traduction) ${ }^{4}$. Les unités JE et MAISON, une fois utilisées en situation, sont bien indexicales, étant donné que leur sens peut varier en fonction du contexte de production et d'interprétation, mais seule l'unité JE est fondamentalement indexicale et constitue par conséquent un déictique appartenant à la deixis du

journal est dite multimodale, au même titre qu'un documentaire ou une interaction médiée par ordinateur ou en face à face.

${ }^{3}$ C'est ce qui permet au post-it « je rends la monnaie », collé sur un distributeur de boissons, de fonctionner sans peine : le JE colle à son référent et instancie le distributeur en tant que locuteur (l'exemple est repris de Charolles, 2002).

${ }^{4}$ « Deixis [...] is the single most obvious way in which the speech setting is embedded in language structure itself » (Hanks, $2005: 191)$. Dans les mots de Fillmore (1975:39), « deixis is the name given to those formal properties of utterances which are determined by, and which are interpreted by knowing, certain aspects of the communicative act in which the utterances in question can play a role ». 
français, car il est impossible de donner une définition hors contexte et intelligible de JE qui ne contienne en son sein une variable contextuelle relative à la situation d'énonciation. Le présent volume s'intéresse donc à la deixis en français en tant qu'elle constitue sa part d'indexicalité lexicalisée, inscrite en langue.

(ii) Plus précisément, le volume se concentre sur ce qui est usuellement appelé la deixis « exophorique », pointant vers un extérieur du dit, c'est-à-dire vers la situation d'énonciation en tant qu'elle constitue les conditions non linguistiques immédiates de l'énoncé : les personnes, le temps, l'espace. La deixis exophorique diffère de la deixis "endophorique», qui pointe du dit, qu'il soit passé ou à venir ( « discourse deixis » chez Fillmore, 1975 ; « textual deixis » chez Lyons, 1977) et que la perspective localisante, qu'on adopte ici, traite sous la dénomination d' « anaphore » (voir Kleiber, 1991, pour une mise au point). Ainsi, dans l'exemple suivant, deux amies discutent en tête-à-tête ${ }^{5}$ :

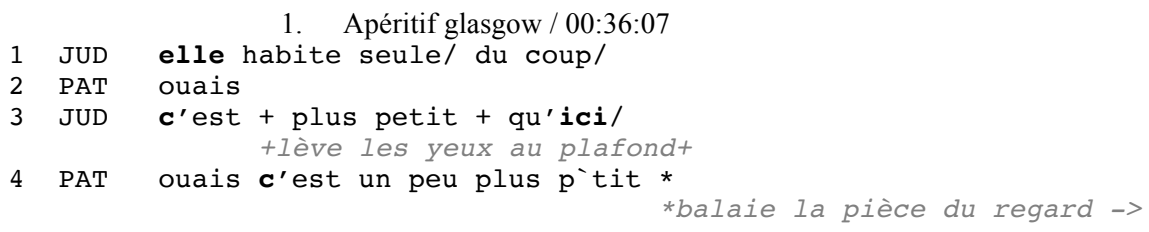

À la ligne 1, le ELLE de « elle habite seule » est anaphorique dans la mesure où la locutrice fait référence à une tierce personne, absente de la situation d'énonciation. Quand bien même l'antécédent ne se situe pas dans l'extrait, la ratification de PAT en 2 signale le caractère bien établi et partagé du référent. Les deux ÇA intégrant les expressions « c'est» des lignes 3 et 4 constituent également des anaphoriques, en relation au fait d' «habiter» de la ligne 1 et au «studio» mentionné quelques secondes avant l'extrait cité. L'accessibilité du référent de ces trois anaphoriques dépend ainsi du cotexte, en d'autres termes des expressions linguistiques utilisées en amont et supposément gardées en mémoire.

Il en va différemment du déictique ICI (ligne 3), dont la référence dépend non de ce qui a été dit précédemment mais de la localisation spatiale de la locutrice. Le fait que le référent du déictique soit à aller chercher dans le contexte immédiat de production est corroboré au plan mimo-gestuel : l'énonciation du ICI est précédée d'un changement de direction du regard, de l'allocutaire au plafond de l'appartement où se trouvent les deux participantes, tandis que la reprise de la comparaison « un peu plus petit [qu'ici]» par PAT en ligne 4 est suivie d'un balayage de la pièce du regard. ICI vient donc embrayer l'énoncé sur son contexte d'énonciation, qui lui donne en retour son ancrage et ses conditions d'intelligibilité. La découverte et la prise en compte de telles unités a eu, comme on va le voir, des conséquences importantes pour la description des formes linguistiques et des pratiques discursives, notamment en langue française.

\section{LE DESTIN DE LA DEIXIS EN LINGUISTIQUE FRANÇAISE}

Le premier point à souligner est l'influence déterminante du travail d'É. Benveniste dans l'émergence d'une réflexion linguistique sur la deixis en français. Avec sa description de «la nature des pronoms» (1966) et de «l'appareil formel de l'énonciation» (1970), É. Benveniste a de fait invité les sciences du langage à un renouvellement de la description linguistique. Si le linguiste s'inscrit dans la démarche saussurienne en tant que celle-ci vise à dégager le caractère systématique des unités au sein d'un tout structuré (une langue), il se propose toutefois d'en considérer un soussystème qui ne se confond pas avec les paradigmes usuellement décrits, tels que les systèmes phonologiques, morphologiques ou encore syntaxiques. É. Benveniste observe en effet que les langues sont organisées, de manière transversale, à un tout autre niveau, celui de la communication, de la parole, ou, selon les mots de l'auteur, en vue de «l'énonciation» en tant qu'elle constitue

${ }^{5}$ L'extrait est tiré de la base CLAPI du laboratoire ICAR (http://clapi.univ-lyon2.fr). «/ » marque une intonation montante et les «+» et «*» délimitent les phénomènes mimo-gestuels (Mondada, 2007). 
«cette mise en fonctionnement de la langue par un acte individuel d'utilisation » (1970 : 12). Faisant écho à l' « origo » déictique décrite par K. Bühler $(1934)^{6}$, É. Benveniste dégage JE, ICI et MAINTENANT comme constituant le centre de repérage vis-à-vis duquel s'organise l'ensemble des déictiques du français. Ainsi, des unités comme DEMAIN, TU ou encore LÀ-BAS font système dans la mesure où il s'agit de « shifters » ou " embrayeurs » (Jakobson, 1957 ; Jespersen, 1922), c'est-à-dire d'«éléments qui réfléchissent l'acte d'énonciation, et qui n'ont de sens que compte tenu des protagonistes et de la situation» (Arrivé, Gadet \& Galmiche, 1986 : 254-255). Sans en parler exactement en ces termes, É. Benveniste (1970) met alors en évidence le caractère « instructionnel» de ces unités particulières (voir la discussion dans Kleiber, 1997), qui invitent le récepteur à aller chercher le référent dans le contexte immédiat de production, et à le faire presque directement, c'est-à-dire sans médiation par le contenu «descriptif» ou conceptuel de l'unité (à distinguer du lexème CHAISE par exemple). Ces unités perturbent ainsi l'opposition entre langue et parole et entre sémantique et pragmatique (Levinson, 2006), dans la mesure où le sémantisme d'une unité comme JE ne peut être établi sans réflexion pragmatique, ou, en termes plus philosophiques, une telle unité ne peut être traitée — tout comme les actes de langage — dans une approche strictement vériconditionnelle de la référence.

Le destin des notions d'énonciation et de deixis en linguistique française se caractérise ensuite par l'émergence de deux directions principales, certes complémentaires, mais toutefois bien distinctes.

(i) Une intégration de l'énonciation et de la deixis en tant que module ou dimension d'appareils théoriques et analytiques plus larges, dont l'interactionisme socio-discursif (Bronckart, 1997), le modèle genevois d'analyse du discours (Roulet, Filliettaz \& Grobet, 2001), la linguistique textuelle (Adam, 2008) ou encore l'analyse du discours à la française (Charaudeau \& Maingueneau, 2002). Pour prendre ce dernier cas, la prise en compte de la deixis sert à l'étude de l'ancrage ou, au contraire, du masquage de la subjectivité non plus dans la langue en général, mais dans certains genres de discours ou types d'interaction, notamment médiatique (e.g. Maingueneau, 1998), politique (e.g. Charaudeau, 2005), littéraire (e.g. Herschberg Pierrot, 1993) ou encore scientifique (e.g. Fløttum, Dahl \& Kinn, 2006).

(ii) Un développement de la linguistique de l'énonciation en tant que réflexion autonome (voir déjà Todorov, 1970 ; puis Culioli, 1990). En guise d'exemples typiques de phénomènes généraux dont l'étude s'inscrit dans une réflexion énonciative portant une attention particulière à la deixis, on peut citer la subjectivité dans le langage (Kerbrat-Orecchioni, 1980), la polyphonie (Bres et al., 2005 ; Ducrot, 1984 ; Nølke, 2001), le discours rapporté (Munoz, Marnette \& Rosier, 2004 ; Rosier, 1999) ou représentation du discours autre (Authier-Revuz, 1995), et plus généralement les ressources et procédés relevant de la prise en compte / prise en charge énonciatives (Coltier, Dendale \& De Brabanter, 2009 ; Laurendeau, 1989).

Cette diversité de perspectives se cristallise dans un ouvrage collectif codirigé par M.-A. Morel et L. Danon-Boileau (1992) qui réunit une cinquantaine de contributions variant au niveau des approches adoptées (diachroniques, synchroniques, comparatistes, psycholinguistiques, discursives), des langues étudiées (avec un accent particulier mis sur le français) et des types de phénomènes indexicaux considérés (temporalité, spatialité, subjectivité). Si on laisse de côté quelques allusions très ponctuelles, aucune contribution ne considère toutefois le rapport de la deixis à la communication non verbale et seule J.-M. Barbéris (1992) étudie un phénomène indexical tel qu'il apparaît à l'oral et dans l'interaction (voir déjà 1987, 1989).

Il faut attendre le début des années 2000 pour qu'émergent différents travaux qui intègrent une composante multimodale dans l'analyse de la deixis en tant qu'elle participe à l'accomplissement de certaines pratiques discursives, qu'il s'agisse de communication politique télévisée (Calbris, 2003) ou de séquences d'explication d'itinéraires (Barbéris, 2008 ; Barbéris \& Manes Gallo, 2007 ; Cosnier, 2007) ${ }^{7}$. Du côté des travaux sur l'acquisition, A. Morgenstern (2006) étudie l'émergence

\footnotetext{
${ }^{6}$ Fillmore (1975) parle quant à lui de « deictic center » et Lyons (1977) de « zero-point ». Sur le rapport entre Bühler et Benveniste, voir Lösener (2010).

${ }^{7}$ Citons encore la synthèse proposée par Charolles sur la référence en français (2002), à l'occasion de laquelle l'auteur réinterroge ponctuellement des fondamentaux de la deixis à l'aune d'hypothèses
} 
et la stabilisation progressives de l'auto-désignation et du déictique JE chez le très jeune enfant, tandis que J.-M. Colletta (2004) rend compte plus généralement du développement du langage verbal et mimo-gestuel - dont l'usage des déictiques — chez l'enfant de six à onze ans. Quant aux travaux de linguistique interactionnelle d'inspiration ethnométhodologique de L. Mondada (2005, 2012, 2015), ils portent une attention particulière à la constitution située, interactionnelle et multimodale - et non plus logo- et égocentrée - de l'origo déictique ${ }^{8}$.

\section{L'ANALYSE MULTIMODALE DE LA DEIXIS DANS LA PAROLE-EN-INTERACTION}

Dans la continuité de ces recherches se développe depuis quelques années une série de travaux visant non seulement à considérer l'articulation d'unités verbales et mimo-gestuelles dans l'accomplissement de pratiques complexes (voir également Hindmarsh \& Heath, 2000), mais aussi à tirer de ces considérations multimodales de quoi problématiser et réinterroger des unités relevant de la deixis au sein d'une langue particulière (notamment l'allemand dans le cas de Fricke, 2012b et de Stukenbrock, 2015). Proposant la notion de «chambre de compression multimodale » [Multimodaler Verdichtungsraum], A. Stukenbrock (2015) identifie des phénomènes de concentration et d'articulation locales et ponctuelles d'unités déictiques et de conduites mimogestuelles, tandis que L. Mondada (2014) commente la possibilité de figements de telles agglutinations, sous la forme de «Gestalts multimodales complexes» [Complex Multimodal Gestalts], ressources sémiotiques composites non réductibles aux seuls déictiques verbaux ni à la relation qu'ils sont supposés entretenir entre eux. Ces travaux participent plus généralement des différentes tentatives qui, depuis plus d'une vingtaine d'années (Duranti \& Goodwin, 1992), décrivent la relation mutuellement constitutive et déterminative qu'entretiennent les ressources de l'action avec leur contexte d'utilisation, qui se trouve donc être à la fois indexé et élaboré par ces mêmes ressources. Et ces recherches tendent à montrer que cette élaboration située, méthodique et négociée du contexte repose sur des pratiques multimodales au sein desquelles la deixis joue un rôle central, en tant qu'elle sert à sélectionner, dans les circonstances ou la situation d'énonciation, les dimensions et éléments pertinents du contexte.

L'observation de telles «configurations séquentielles multimodales» (Traverso, 2016) invite donc à décrire d'abord l'acte de référence, tel qu'accompli de manière potentiellement multimodale dans la parole-en-interaction, pour en considérer ensuite la dimension linguistique et en dégager une analyse des déictiques verbaux à l'œuvre. L'originalité du présent volume réside ainsi dans l'ambition d'extraire du passage par la multimodalité et par le travail sur corpus vidéo-enregistré de quoi contextualiser le fonctionnement de ces unités linguistiques spécifiques que sont les déictiques. Dit autrement, le volume cherche à adopter une posture réflexive sur les unités appartenant à la deixis française du moment où la multimodalité de la référence est non seulement prise en compte, mais aussi intégrée à la démarche théorique et analytique. En termes ethnométhodologiques, il s'agit d'opérer une « re-spécification » (Garfinkel, 1991), c'est-à-dire de repartir de certaines évidences, dans le cas présent relativement à la deixis et aux déictiques, en les réinterrogeant à l'aune de leur émergence et fonctionnement en milieu naturel, fondamentalement multimodal ou du moins plurisémiotique. Interroger l'ancrage multimodal de la deixis et en évaluer les conséquences pour l'appréhension de certains déictiques du français revient à poursuivre l'objectif formulé par G. Kleiber (1991 : 16) sur la base d'une réflexion de F. Corblin : « dire que A est déictique est en somme bien trop vague : il faudrait se donner les moyens de dire en quoi A est déictique ». En repartant de la fascination que suscite la deixis élevée au statut de preuve que le système linguistique est le produit de l'usage, il s'agit d'inclure dans la réflexion et dans l'analyse la dimension multimodale de ce dernier.

multimodales. À la différence des travaux réunis dans le cadre du présent volume, les séquences mobilisées par Charolles sont très courtes, non extraites de corpus structurés et souvent inventées.

${ }^{8}$ Klein (1982 : 163-164) évoquait déjà la possibilité, pour un geste produit lors de séquences d'explications d'itinéraires, de créer une origo secondaire suspendant la pertinence de l'origo de base de l'énonciation (voir également Kleiber, 1993, pour un commentaire). 
En poursuivant l'effort d'étudier les ressources linguistiques en contexte, dans le cas présent dans la parole-en-interaction multimodale, le volume alimente le dialogue et valorise la complémentarité entre une linguistique du discours — ou une linguistique des emplois — et une linguistique de la langue - ou une linguistique du système. Il s'agit de chercher ce qui fait système dans l'emploi et ce qui est orienté vers l'emploi dans le système, en abordant les unités linguistiques comme des ressources pour l'action, dont le caractère systématique émerge de l'usage en contexte (voir notamment Auer \& Pfänder, 2011 ; Hopper, 1987).

\section{Présentation du numéro et des contributions}

Le volume réunit sept contributions relevant de l'analyse multimodale de la parole-eninteraction. Il privilégie une approche empirique, dans la mesure où les contributions fondent leurs questions de recherche, analyses et conclusions sur des données vidéo-enregistrées de situations naturelles et constituées en corpus ou collections documentant les phénomènes soumis à l'investigation. Interactionnistes, elles s'efforcent de baser l'analyse et l'interprétation sur les phénomènes de co-orientation tels qu'ils émergent de la dynamique négociative. Elles cherchent à tirer des analyses sur corpus de quoi dialoguer avec les hypothèses et définitions généralement acceptées, notamment dans d'autres traditions de recherche. Les contributions font par ailleurs preuve de complémentarité aux niveaux de la (i) perspective adoptée, du (ii) type de deixis considérée et des (iii) contextes analysés.

(i) Alors que certaines contributions, plus générales, travaillent sur des empans de formes, en cherchant à dégager leur distribution et leur systématicité à partir de leur fonctionnement multimodal (Colletta; Kerbrat-Orecchioni \& Constantin de Chanay), d'autres partent d'un réexamen d'une forme spécifique (Horlacher \& De Stefani ; Jacquin; Mondada ; Morgenstern, Caët \& Limousin ; Ticca, Traverso \& Ursi). Deux contributions enrichissent par ailleurs l'étude de la deixis à partir d'une perspective acquisitionnelle (Colletta ; Morgenstern, Caët \& Limousin).

(ii) Les contributions couvrent la deixis spatiale (Colletta ; Horlacher \& De Stefani), la deixis personnelle (Jacquin; Kerbrat-Orecchioni \& Constantin de Chanay; Morgenstern, Caët \& Limousin) et la deixis temporelle (Colletta ; Horlacher \& De Stefani ; Mondada ; Ticca, Traverso \& Ursi).

(iii) Les contributions documentent et traitent de différents genres interactionnels, notamment des débats publics et/ou télévisés (Jacquin; Kerbrat-Orecchioni \& Constantin de Chanay), des visites guidées (Ticca, Traverso \& Ursi), des interactions de service en salon de coiffure (Horlacher $\&$ De Stefani), des courses automobiles (Mondada) et des contextes conversationnels impliquant des enfants (Colletta ; Morgenstern, Caët \& Limousin).

Résumés ci-dessous, les différents articles témoignent de l'intérêt et de l'apport théoriques et analytiques d'étudier la deixis verbale dans une perspective multimodale, en rendant compte de la relation qui opère entre des ressources linguistiques indexicales et le contexte sémiotiquement riche sur lequel celles-ci sont indexées. Premièrement, les contributions prennent acte et rendent compte de la multimodalité des pratiques langagières en situation de co-présence, qu'il s'agisse de pratiques descriptives, narratives, argumentatives ou encore instructionnelles. Deuxièmement, elles permettent de situer la deixis au sein de la diversité des ressources référentielles mobilisées par ces pratiques multimodales. Elles problématisent ainsi les frontières de la deixis, notamment vis-à-vis de l'anaphore, ainsi que la dimension indexicale de déictiques verbaux classiquement étudiés. Elles montrent notamment que la mimo-gestualité contribue à désambiguïser et sélectionner entre les différentes valeurs d'un déictique verbal, à spécifier une de ses valeurs potentielles ou encore à autoriser la superposition de différentes valeurs qui pouvaient sembler incompatibles ou exclusives au premier abord. Troisièmement, et ce faisant, elles éclairent d'un jour nouveau la polysémie et la polyfonctionnalité des déictiques, en décrivant leur émergence au sein de ressources référentielles indexicales et multimodales (qu'on les appelle «chambres de compression», «Gestalts » ou « configurations »).

A.-S. Horlacher \& E. De Stefani reviennent sur le déictique LÀ dont ils étudient l'ancrage multimodal et les différents emplois dans un corpus vidéo-enregistré d'interactions professionnelles en salon de coiffure. Si la complexité de LÀ est bien documentée, l'originalité de la contribution 
tient dans la prise en compte de la dimension à la fois située, interactionnelle et multimodale des pratiques référentielles considérées. En adoptant cette perspective praxéologique, les auteurs montrent que la présence de gestes de pointage ou de préhension cooccurrents participe de l'interprétation de LÀ en tant que déictique spatial, tandis que leur absence tend à favoriser une lecture temporelle ou anaphorique. En commentant différentes possibilités d'articulation entre une ressource linguistique plastique et certaines conduites mimo-gestuelles spécifiques, la contribution problématise la polyfonctionnalité de la ressource, notamment la possibilité pour LÀ de servir à la fois la construction d'une attention conjointe, la référenciation extralinguistique et la mise en contraste.

La contribution de L. Mondada questionne pour sa part le déictique temporel dont on pourrait penser de prime abord qu'il constitue le plus évident, le moins problématique, du moins le moins sujet à une respécification multimodale: MAINTENANT. Au travers d'une étude relevant de l'analyse conversationnelle d'inspiration ethnométhodologique, l'auteure problématise la coïncidence entre origo temporelle et production de MAINTENANT dans des contextes où la succession rapide d'actions constitue une condition de bon accomplissement de l'activité et où cette coïncidence fait ainsi l'objet d'un travail méticuleux de préparation. Plus concrètement, L. Mondada étudie MAINTENANT en fin d'acte directif énoncé par un instructeur à destination d'un pilote de course (« et là tu braques euh maintenant»). L'auteure montre le haut degré d'indexation du MAINTENANT sur l'activité multimodale, tant au niveau de la forme (raccourcie, accélérée) du déictique que de sa valeur compte tenu de sa position séquentielle et de son environnement multimodal. Le tout fait de ce MAINTENANT un moyen de construire une origo temporelle très ponctuelle et inscrite dans une « Gestalt multimodale » particulière, instructionnelle.

A.-C. Ticca, V. Traverso \& B. Ursi se concentrent quant à eux sur TOUT À L'HEURE en tant qu'unité déictique distincte et complémentaire de MAINTENANT : ambigu, TOUT À L'HEURE permet de référer à un moment situé soit dans une antériorité soit dans une postériorité relativement au moment d'énonciation. Partant d'un corpus de visites guidées vidéo-enregistrées, les auteurs soulignent la polyfonctionnalité de la ressource, qui intègre des effets d'annonce quant à la trajectoire future de la visite (« ce qu'on verra tout à l'heure »), des transitions d'un lieu/objet visité à un autre («tout à l'heure on a été dans $X »)$ ou encore des incises explicatives ou métadiscursives («ce que je vous disais tout à l'heure »). Si le rôle de la mimo-gestualité est accessoire dans la désambiguïsation entre ces différentes valeurs, A.-C. Ticca, V. Traverso \& B. Ursi dévoilent des phénomènes de cooccurrence intéressants entre la ressource et son ancrage multimodal. Tendanciellement, TOUT À L'HEURE n'est pas accompagné de pointages mimogestuels ou posturaux précis et saillants. Cela semble s'expliquer par le fait qu'il s'agit pour les guides d'établir une mise en contraste entre le [LÀ-BAS - TOUT À L'HEURE] et le [ICI MAINTENANT], en se focalisant sur le second, qui est multimodalement élevé comme objet de l'attention conjointe à établir au moment de l'énonciation. Les quelques cas de pointage mimogestuel précis accompagnés d'un changement de direction du regard sont réservés à la référence au passé, probablement du fait de l'accessibilité partagée du référent entre les guides et les visiteurs.

La contribution de J. Jacquin revient sur le pronom ON, dont la complexité sémantique et référentielle a souvent été soulignée : ON constituerait une ressource d'anonymisation dans la mesure où l'utiliser reviendrait à substituer une ressource à portée référentielle plus spécifique (ON pour JE, pour TU, pour DES GENS, etc.). À partir d'une analyse énonciative et multimodale d'extraits de débats publics et télévisés, la contribution questionne la dimension indexicale du pronom en tant qu'il émerge de conduites multimodales particulières. L'auteur montre la possibilité d'une coexistence des valeurs indéfinie, orientée vers la décontextualisation et la généralisation, et définie, orientée vers la contextualisation et la spécification. Cette coexistence répond à la nécessité pour le débattant d'attaquer une position idéologique générale tout en l'indexant sur l'adversaire en tant qu'il ou elle l'incarne, au sens propre. Plutôt qu'une démarche substitutive, consistant à chercher l'équivalent référentiel de ON dans le même paradigme, J. Jacquin plaide, avec d'autres, pour l'option consistant à toujours partir de la valeur fondamentalement indéfinie de ON pour voir 
ensuite comment cette valeur peut être localement aménagée, travaillée, complétée, notamment au niveau multimodal.

H. Constantin de Chanay \& C. Kerbrat-Orecchioni poursuivent leur exploration des six débats présidentiels français d'entre-deux-tours en considérant le rôle du regard dans les phénomènes d'adresse. Une attention particulière est portée aux cas de divergence des indices, c'est-à-dire lorsque les instructions véhiculées respectivement par les formes linguistiques et le comportement mimo-gestuel, et en particulier le regard, sont concurrentes, leur articulation témoignant alors d'enjeux stratégiques cruciaux. Par exemple, un débattant peut utiliser une forme nominale d'adresse délocutive («Monsieur X a dit») tout en regardant, selon les cas, tout aussi bien son adversaire ainsi désigné qu'un animateur. Mêlant observations quantitatives et analyses qualitatives, les auteurs dégagent non seulement des profils de communicateurs politiques différenciés, mais également les types de coordination possible entre les différentes ressources indexicales disponibles. H. Constantin de Chanay \& C. Kerbrat-Orecchioni problématisent ainsi les frontières de la deixis personnelle à partir du rôle joué par le regard.

A. Morgenstern, S. Caët \& F. Limousin étudient, en les comparant, les ressources d'autodésignation telles qu'elles émergent progressivement chez deux enfants dans leur acquisition respective de la langue française et de la langue des signes française. En tant que renvoyant par excellence à l'origo déictique, on pourrait croire que ces unités gravitant autour du JE de l'énonciation sont les plus nécessaires à l'élaboration du reste du système déictique et par conséquent qu'elles apparaissent tôt chez l'enfant. On sait pourtant que ce n'est pas le cas, dans la mesure où une unité comme JE exige d'intégrer le principe de réversibilité des pronoms. On pourrait alors supposer qu'il en va différemment pour l'enfant malentendant, dans la mesure où l'auto-pointage peut sembler plus motivé, plus transparent qu'une unité comme JE. Les auteures montrent qu'il n'en est rien : qu'il soit socialisé en langue française ou en langue des signes française, l'enfant commence par des énoncés rhématiques à un terme («manger »), où l'autodésignation est implicite et reconstructible contextuellement à partir de l'environnement multimodal de l'énoncé (direction des gestes, postures, mises en situation pratique), avant d'élaborer progressivement, notamment du fait des contraintes syntaxiques de la langue cible, des énoncés à deux termes où le sujet grammatical est rempli par un déictique d'auto-désignation («je / auto-pointage $»+«$ manger $»)$.

Finalement, la contribution de J.-M. Colletta sur la deixis spatiale boucle le parcours, en réinterrogeant les frontières de la référence indexicale à partir de corpus documentant différentes activités langagières accomplies par des enfants de tous âges. L'auteur en extrait des séquences où il étudie l'articulation entre les déictiques verbaux typiques de la deixis spatiale et objectale (e.g. ICI, LÀ-BAS, ÇA, CE, CELUI-LÀ) et des pointages mimo-gestuels mobilisant différentes parties du corps (doigt, main, tête, buste). La prise en compte de la mimo-gestualité dans les phénomènes référentiels met à jour la présence d'un gradient, allant du plus indexical ou « exophorique », c'està-dire une référence via un ancrage dans le contexte perceptuel immédiat des interlocuteurs, au plus représentationnel ou " endophorique », c'est-à-dire une référence via une abstraction du contexte immédiat et un transfert dans un domaine imaginé, raconté ou conceptuel. Les exemples témoignent ainsi de la richesse et de la complexité de l'accessibilité des référents en situation multimodale ${ }^{9}$.

\footnotetext{
${ }^{9}$ L'auteur tient à remercier Jacques Bres et Catherine Kerbrat-Orecchioni pour leur relecture précieuse d'une première version de ce texte. Il garde néanmoins la responsabilité de toutes les maladresses et imprécisions qui subsisteraient.
} 


\section{Références}

ADAM J.-M. (2008), La linguistique textuelle: introduction à l'analyse textuelle des discours [2ème édition]. Paris : Armand Colin.

Almeida M. E. (2000), La deixis en portugais et en français. Leuven : Peeters.

Arrive M., Gadet F. \& Galmiche M. (1986). La Grammaire d'Aujourd'hui. Paris : Flammarion.

Auer P. (1988), “On Deixis and Displacement”, Folia Linguistica 22(3-4), 263-292.

AuER P. \& PFÄNDER S. (eds.) (2011), Constructions. Emerging and Emergent. Berlin: Mouton de Gruyter.

Authier-Revuz J. (1995), Ces mots qui ne vont pas de soi. Boucles réflexives et noncoïncidences du dire (Vol. 1-2). Paris : Larousse.

BARberis J.-M. (1987), « Deixis spatiale et interaction verbale : un emploi de "là" », Cahiers de praxématique $9,23-48$.

BARBERIS J.-M. (1989), « Deixis et balisage du parcours narratif: le rôle pivot de l'adverbe "là" dans des récits de lutte ", Langages 93, 45-63.

BARBERIS J.-M. (1992), «Un emploi déictique propre à l'oral : le "là" de clôture, in M.-A. Morel \& L. Danon-Boileau (éds.), La deixis. Colloque en Sorbonne (8-9 juin 1990), Paris : PUF, 567-578.

BARBERIS J.-M. (2008), «La deixis spatiale dans les descriptions d'itinéraires piétons: comment s'orienter dans l'espace de la ville », in M. Vuillaume (éd.), Ici et maintenant, Amsterdam : Rodopi, 199-219.

Barberis J.-M. \& Manes Gallo M. C. (éds.) (2007), Parcours dans la ville. Description d'itinéraires piétons, Paris : L'Harmattan.

Benveniste E. (1966), «La nature des pronoms », in Problèmes de linguistique générale (Tome 1), Paris : Gallimard, 251-257.

Benveniste E. (1970), «L'appareil formel de l'énonciation », Langages 17, 12-18.

BerRendonNer A. (1981), Éléments de pragmatique linguistique, Paris : Minuit.

Bres J., Haillet P. P., Mellet S., Nølke H. \& Rosier L. (éds.) (2005), Dialogisme et polyphonie: approches linguistiques, Bruxelles : De Boeck Université.

BRONCKART J.-P. (1997), Activité langagière, textes et discours: pour un interactionisme socio-discursif, Lausanne/Paris : Delachaux et Niestlé.

BÜHLER K. (1934), Sprachtheorie. Die Darstellungsfunktion der Sprache, Stuttgart: Fischer.

CALBRIS G. (2003), L'expression gestuelle de la pensée d'un homme politique, Paris : CNRS Editions.

Charaudeau P. (2005), Le discours politique : les masques du pouvoir, Paris : Vuibert.

Charaudeau P. \& Maingueneau, D. (éds.) (2002), Dictionnaire d'analyse du discours, Paris : Seuil.

Charolles M. (2002), La référence et les expressions référentielles en français, Paris : Ophrys.

Colletta J.-M. (2004), Le développement de la parole chez l'enfant âgé de 6 à 11 ans : corps, langage et cognition, Hayen : Mardaga.

Coltier D., Dendale P. \& De Brabanter P. (2009), « La notion de prise en charge : mise en perspective », Langue française 162, 3-27. 
COSNIER J. (2007), «Les gestes de la transmission d'itinéraires entre piétons », in J.-M. Barbéris \& M. C. Manes Gallo (éds.), Parcours dans la ville. Descriptions d'itinéraires piétons, Paris : L'Harmattan, 230-239.

Culioli A. (1990), Pour une linguistique de l'énonciation. Opérations et représentations (Tome 1), Paris : Ophrys.

Deppermann A. (ed.) (2013), Conversation Analytic Studies of Multimodal Interaction / Journal of Pragmatics 46(1).

Ducrot O. (1984), Le dire et le dit, Paris : Minuit.

Duranti A. \& Goodwin C. (eds.) (1992), Rethinking Context: Language as an Interactive Phenomenon, Cambridge: Cambridge University Press.

EHRICH V. (1992), Hier und jetzt : Studien zur lokalen und temporalen Deixis im Deutschen, Tübingen: Niemeyer.

Fillmore C. J. (1975), Santa Cruz Lectures on Deixis 1971, Bloomington: Indiana University Linguistics Club.

Fløtтum K., Dahl T. \& KinN T. (2006), Academic Voices: Across Languages and Disciplines, Amsterdam: John Benjamins.

FRICKE E. (2012a), Grammatik multimodal: Wie Wörter und Gesten zusammenwirken, Berlin: Walter de Gruyter.

Fricke E. (2012b), Origo, Geste und Raum, Lokaldeixis im Deutschen, Berlin, Boston: De Gruyter.

GARFINKEL H. (1967), Studies in ethnomethodology, Englewood Cliffs (N.J.): Prentice-Hall.

GARFINKEL H. (1991), "Respecification: evidence for locally produced, naturally accountable phenomena of order, logic, reason, meaning, method, etc. in and as of the essential haecceity of immortal ordinary society (I) - an announcement of studies", in G. Button (ed.), Ethnomethodology and the human sciences, Cambridge: Cambridge University Press, 10-19.

Goodwin C. (1987), «La référence exophorique comme procédé interactif», Cahiers de praxématique $9,9-22$.

HANKS W. F. (2005), "Explorations in the Deictic Field", Current Anthropology 46(2), 191-220.

HAUSENDORFF H. (1995), "Deixis and Orality: Explaining Games in Face-to-Face Interaction”, in U. M. Quastoff (ed.), Aspects of Oral Communication, Berlin: Walter de Gruyter, 181-197.

Herschberg Pierrot A. (1993), Stylistique de la prose, Paris : Belin.

Hindmarsh J. \& HeAth C. (2000), "Embodied reference: A study of deixis in workplace interaction", Journal of Pragmatics 32, 1855-1878.

HOPPER P. (1987). Emergent Grammar, in Proceedings of the Thirteenth Annual Meeting of the Berkeley Linguistics Society, 139-157.

JAKOBSON R. (1957), Shifters, Verbal Categories, and the Russian Verb, Cambridge, Harvard University: Depart. of slavic languages and literatures.

Jeppesen Kragh K. \& Lindschouw J. (eds.) (2013), Deixis and pronouns in romance languages, Amsterdam: John Benjamins.

JESPERSEN O. (1922), Language. Its Nature, Development and Origin, London: George Allen \& Unwin, ltd.

JEwITT C. (Éd.) (2009), Handbook of Multimodal Analysis, London: Routledge. 
Jones S. E. \& LeBARON C. D. (2002), "Research on the Relationship Between Verbal and Nonverbal Communication: Emerging Integrations", Journal of Communication 52(3), 499-521.

Kerbrat-Orecchioni C. (1980), L'énonciation. De la subjectivité dans le langage, Paris : Armand Colin.

KLEIBER G. (1991), « Anaphore-deixis : où en sommes-nous ? », L'information grammaticale 51, 3-18.

KLEIBER G. (1993), «L'espace d'ICI : sur la pragma-sémantique des adverbes spatiaux. Le cas d'Il fait chaud ici », Cahiers de linguistique française 14, 85-104.

KLEIBER G. (1997), «Sens, référence et existence: que faire de l'extra-linguistique? », Langages 127, 9-37.

KLEIN W. (1982), "Local Deixis in Route Directions", in R. J. Jarvella \& W. Klein (eds.), Speech, Place, and Action, New York: Wiley \& Sons, 161-182.

Kress G. (2009), "What is mode", in C. Jewitt (ed.), Handbook of Multimodal Analysis, London: Routledge, 54-67.

Kress G. \& VAn Leeuwen, T. (2001), Multimodal Discourse, London: Arnold.

LAURENDEAU P. (1989), « Repérage énonciatif et valeur de vérité : la prise en compte, la prise en charge », in D. Vincent (éd.), Des analyses de discours, Québec : Université Laval, 107-129.

LENZ F. (ed.) (2003), Deictic Conceptualisation of Space, Time and Person, Amsterdam: John Benjamins.

LEVINSON S. C. (2006), "Deixis", in L. R. Horn \& G. Ward (eds.), The Handbook of Pragmatics, Malden, MA \& Oxford: Blackwell Publishing, 97-121.

LÖSENER H. (2010), "Die Origo der Subjektivität: ich, jetzt, hier bei Bühler und Benveniste", in A. ten Cate, R. Rapp, J. Strässler, M. Vliegen, H. Weber \& W. Kürschner (eds.), Grammatik - Praxis - Geschichte. Festschrift für Wilfried Kürschner, Tübingen: Narr, 155-165.

LyONS J. (1977), Semantics (Vol. 2), Cambridge: Cambridge University Press.

Maingueneau D. (1998), Analyser les textes de communication, Paris : Dunod.

MONDADA L. (2005), « La constitution de l'origo déictique comme travail interactionnel des participants : une approche praxéologique de la spatialité », Intellectica 2/3(41-42), $75-100$.

Mondada L. (2007). "Multimodal Resources for Turn-Taking: Pointing and the Emergence of Possible Next Speakers", Discourse Studies 9(2), 194-225.

MondadA L. (2012), "Deixis: An Integrated Interactional Multimodal Analysis", In P. Bergmann, J. Brenning, M. Pfeiffer \& E. Reber (eds.), Prosody and Embodiment in Interactional Grammar, Berlin, Boston: De Gruyter, 173-206.

MondadA L. (2014), “The Local Constitution of Multimodal Resources for Social Interaction", Journal of Pragmatics 65, 137-156.

Mondada L. (2015), "Social Interaction”, in K. Jungbluth \& F. D. Milano (eds.), Manual of Deixis in Romance Languages, Berlin, Boston: De Gruyter, 661-683.

Morel M.-A. \& Danon-Boileau, L. (éds.) (1992), La deixis. Colloque en Sorbonne (8-9 juin 1990), Paris : PUF.

Morgenstern A. (2006), Un JE en construction. Genèse de l'auto-désignation chez le jeune enfant, Paris : Ophrys. 
MORRIS C. W. (1938), "Foundations of the Theory of Signs", in O. Neurath, R. Carnap \& C. W. Morris (éds.), Foundations of the Unity of Science. Toward an International Encyclopedia of Unified Science, Chicago: University of Chicago Press, 77-137.

Müller C., Cienki A., Fricke E., Ladewig S. H., McNeill D. \& Tessendorf S. (éds.) (2013), Body - Language - Communication. An International Handbook on Multimodality in Human Interaction [Vol.1], Berlin: Mouton de Gruyter.

Munoz J. M. L., MARnetTe S. \& Rosier L. (éds.) (2004), Le discours rapporté dans tous ses états, Paris : L'Harmattan.

NøLKe H. (2001), Le regard du locuteur 2 : pour une linguistique des traces énonciatives, Paris : Kimé.

NORRIS S. (2004), Analyzing multimodal interaction: a methodological framework, New York: Routledge.

PeirCe C. S. (1932), Collected Papers / Vol. 2 Elements of Logic (C. Hartshorne \& P. Weiss, Éds.), Cambridge: Harvard University Press.

Perkins R. D. (1992), Deixis, grammar, and culture, Amsterdam: John Benjamins.

Rosier L. (1999), Le discours rapporté : histoire, théories, pratiques, Bruxelles : De Boeck Université.

Roulet E., Filliettaz L. \& Grobet A. (2001), Un modèle et un instrument d'analyse de l'organisation du discours, Berne : Peter Lang.

SCHMAUKS D. (1991), Deixis in der Mensch-Maschine-Interaktion: multimediale Referentenidentifikation durch natürliche und simulierte Zeigegesten, Tübingen: Niemeyer.

SEnFT G. (Éd.) (2004), Deixis and demonstratives in Oceanic languages, Canberra: Pacific Linguistics.

Sidnell J. \& Stivers T. (eds.) (2005), Multimodal Interaction / Semiotica 156, Berlin: Walter de Gruyter.

Sidnell J. \& STIVERs T. (eds.) (2013), The Handbook of Conversation Analysis, Chichester: Wiley-Blackwell.

Stukenbrock A. (2015), Deixis in der face-to-face-Interaktion, Berlin: Mouton De Gruyter.

Suchman L. (1987), Plans and Situated Actions. The Problem of Human-Machine Communication, Cambridge: Cambridge University Press.

TAnaKa S. (2011), Deixis und Anaphorik, Referenzstrategien in Text, Satz und Wort, Berlin, Boston: De Gruyter.

Todorov T. (éd) (1970), L'énonciation / Langages 17, Paris : A. Colin.

Traverso V. (2016), Décrire le français parlé en interaction, Paris : Ophrys. 